\title{
Preconception Care and Treatment with Assisted Reproductive Technologies
}

\author{
David A. Grainger • Linda M. Frazier • \\ Courtney A. Rowland
}

Published online: 27 June 2006

(C) Springer Science+Business Media, Inc. 2006

\begin{abstract}
Couples with fertility problems seeking treatment with assisted reproductive technologies (ART) such as in vitro fertilization should receive preconception counseling on all factors that are provided when counseling patients without fertility problems. Additional counseling should address success rates and possible risks from ART therapies. Success rates from ART are improving, with the highest live birth rates averaging about $40 \%$ per cycle among women less than 35 years old. A woman's age lowers the chance of achieving a live birth, as do smoking, obesity, and infertility diagnoses such as hydrosalpinx, uterine leiomyoma, or male factor infertility. Singletons conceived with ART may have lower birth weights. Animal studies suggest that genetic imprinting disorders may be induced by certain embryo culture conditions. The major risk from ovarian stimulation is multiple gestation. About one-third of live-birth deliveries from
\end{abstract}

D. A. Grainger · L. M. Frazier · C. A. Rowland

Department of Obstetrics and Gynecology, University of Kansas

School of Medicine-Wichita,

Wichita, Kansas

D. A. Grainger · L. M. Frazier

Women's Research Institute,

Wichita, Kansas

D. A. Grainger · L. M. Frazier

Department of Preventive Medicine and Public Health, University of Kansas School of Medicine-Wichita,

Wichita, Kansas

L. M. Frazier $(\bowtie)$

Departments of Obstetrics and Gynecology and Preventive

Medicine and Public Health, University of Kansas School of

Medicine-Wichita,

1010 N. Kansas Avenue, Wichita, KS, 67214

e-mail: 1frazier@kumc.edu
ART have more than one infant, and twins represent $85 \%$ of these multiple-birth children. There are more complications in multiple gestation pregnancies, infants are more likely to be born preterm and with other health problems, and families caring for multiples experience more stress. Transferring fewer embryos per cycle reduces the multiple birth rate from ART, but the patient may have to pay for additional cycles of ART because of a lower likelihood of pregnancy.

Keywords Infertility · Preconception counseling Pregnancy complications $\cdot$ Pregnancy $\cdot$ Multiple $\cdot$ in vitro fertilization - Assisted reproductive technologies

\section{Current knowledge}

Fertility problems affect about $10 \%$ of couples in developed countries [1-3]. These couples should receive preconception counseling on all factors that are discussed during preconception counseling for patients without fertility problems. Additional counseling should address success rates and possible risks from the specific fertility treatments being considered because of the couple's diagnoses and preferences. To provide an example of an approach to preconception counseling for the infertile couple, treatment with assisted reproductive technologies (ART) such as in vitro fertilization (IVF) is reviewed here.

About $1 \%$ of births in the United States are the result of ART [4]. ART care represents a unique opportunity for preconception counseling because the moment of conception is easily discernable. Counseling should address expected success rates from ART based on the woman and the man's risk profile. Older maternal age is the strongest risk factor for not achieving a successful outcome from ART. Live birth rates average about $40 \%$ per cycle among women less than 
35 years old, and gradually decrease to about $6 \%$ per cycle among women over age 42 who use their own oocytes [4]. Older women who accept donor oocytes increase their chance of a live birth to approximately the rate seen in the age group of the donor woman [5]. Cumulative live birth rates account for the fact that many couples return for further treatment if the first cycle of ART is unsuccessful. For couples who complete two to three ART cycles, cumulative live birth rates are about $50-60 \%$ for women 35 years old or younger, declining to about $30 \%$ by age 40 [6]. As men's age increases, the time required for a couple to conceive lengthens, even after controlling for the woman's age and other risk factors for reduced fertility [7].

The chance of having a live birth from ART therapy is influenced by the health habits and the infertility diagnoses of the couple. Current tobacco smoking by women decreases ovarian function and is manifested by increased basal levels of follicle stimulating hormone. Such women produce fewer oocytes during ART and have lower pregnancy rates $[8,9]$. Current smoking by the male partner also decreases pregnancy rates through direct effects on sperm and by exposing the woman partner to side-stream smoke. Increasing body mass index (BMI) is associated with decreasing ovarian response to follicle stimulating hormone, resulting in fewer oocytes collected in a given IVF cycle, as well as increasing rates of early pregnancy loss [10]. Although no strong evidence associates underweight with adverse ART outcomes, maternal underweight (BMI $<18.5 \mathrm{~kg} / \mathrm{m}^{2}$ ) is risk factor for low birth weight in the general population.

As the number of specific infertility diagnoses increases for a couple, pregnancy and live birth rates from ART decrease. Moreover, certain infertility diagnoses are somewhat more difficult to overcome than others during treatment. Live birth rates after ART tend to be slightly higher if the only diagnosis is endometriosis, and slightly lower if the only diagnosis is uterine leiomyoma or hydrosalpinx $[4,6,11]$. Leiomyoma may decrease uterine receptivity, mainly when an intramural or submucosal fibroid distorts the endometrial cavity. The mechanism by which hydrosalpinx worsens the prognosis in ART is not understood, but tubal fluid in hydrosalpinx has embryotoxic properties and salpingectomy improves live birth rates [12]. Male factor infertility is present in about $40 \%$ of couples presenting for ART, and may be a barrier to achieving pregnancy unless intracytoplasmic sperm injection (ICSI) is used [4, 6, 11].

The most common adverse outcome from infertility treatment is multiple gestation. ART produced 11,544 multiple birth deliveries in the United States during 2002 [4]. Of all the ART infants born that year, $45.7 \%$ were twins and $7.6 \%$ were triplets or higher order multiples. Although health outcomes are good to excellent in many twin pregnancies, mothers of twins experience more pregnancy complications, lasting infant neurologic impairment may occur from very preterm birth, and the neonatal death rate of twins is 5-10 times greater than of singletons. For triplets, $90 \%$ of births are preterm and the relative risk of death in the first month of life is increased 20-fold [13].

To place the risk of multiple gestation from ART in perspective, estimates are available on the proportion of multiple births from unassisted conception and from treatment with ovulation medications without ART. These estimates have been made by subtracting the rate of multiple births during the era before ovulation-inducing drugs (unassisted conception) and the rate of multiple births resulting from ART from the total rate of multiple births, adjusted for trends in maternal age $[4,14]$. Such an analysis shows that about $65 \%$ of twins and 15-18\% of triplets are from natural conception each year. About $20 \%$ of twins and $35-40 \%$ of triplets are probably from ovulation induction without ART, and about $15 \%$ of twins and $40-45 \%$ of triplet births result from ART [14].

In general, women with the best prognosis for achieving a live birth from ART are younger than 35 years, use freshly fertilized embryos from their own eggs, and have good quality embryos-a marker of the latter is having more embryos available than are transferred. For this group, recent surveillance statistics showed that transferring one, two, three or four embryos resulted in live birth rates of 47.4, 51.8, 49.6 and $45.5 \%$, respectively; the singleton rates among these births were 100.0, 60.4, 52.8 and $40.7 \%$, respectively [4]. By age 38-40 years the prognosis is poorer among all women who use freshly fertilized embryos from their own eggs. Transferring one, two, three or four embryos in this group resulted in live birth rates of 10.4, 23.3, 28.9 and $29.9 \%$ respectively; the singleton rates among these births were 97.4, $81.2,79.2$ and $70.4 \%$, respectively. Thus, for women with the best prognosis, transferring more embryos provides little improvement in live birth rates with an increasing risk of multiples. For women with a poorer prognosis, additional embryos may improve live birth rates up to a point, and the risk for multiples may increase less dramatically [4].

During ART, ovarian hyperstimulation syndrome is associated with production of a large number of follicles. Usually the only symptoms are modest abdominal pain. More severely affected women, however, experience increased capillary permeability that leads to ascites and in rare cases, death. Symptoms requiring hospitalization occur in $0-3 \%$ of IVF cycles [15]. Ovarian cancer risk from medications used to stimulate folliculogenesis has been studied because of concerns that this treatment may trigger growth of ovarian cortical cells through the process of healing at the sites of ovulation. Although studies show that women treated with ART have about a 50\% increase in ovarian cancer risk compared to the general population, when compared to control women with infertility who did not receive ART, ovarian cancer risk is not elevated [16]. Thus it appears that 
infertility itself may be the risk factor for ovarian cancer, rather than ART.

Singletons conceived with ART may be at greater risk of being born prematurely, or to be small for gestational age [17]. Available studies have been limited by lack of data on key confounders. When matching is used to control for the most important confounders, the risk difference between spontaneously conceived singletons and singletons conceived with ART remains, but appears to be relatively small. The rates of fetal growth restriction differ by about $3.7 \%$, with an overall rate of growth restriction of $8.6 \%$ among spontaneous singletons compared to a rate of $12.3 \%$ among singletons conceived with ART. The rates of very preterm birth differ by about $1.3 \%$, with an overall rate of very preterm birth of $0.7 \%$ for spontaneous singletons compared to $2.0 \%$ for singletons conceived with ART [17].

In some studies, an increased risk of birth defects has been noted among IVF children compared to the general population, but in other studies the difference tends to disappear when risk factors for both infertility and birth defects such as older parental age and multiple gestation are controlled [18]. Infertility is sometimes caused by sex chromosome aberrations, such as $\mathrm{Y}$ chromosome deletions that lead to reduced sperm counts in men $[18,19]$. Sperm of men with reduced fertility has more aneuploidy [20]. Whether treatment of male factor infertility by ICSI will cause fertility problems in male offspring cannot be known until long-term follow-up is available.

Adverse embryo culture conditions can cause imprinting disorders in mice during a procedure similar to ART. This lends biologic plausibility to imprinting disorders as a possible adverse effect of IVF [18, 19, 21]. Imprinting is the mechanism by which congenital disorders such as Beckwith-Wiedemann syndrome, Angelman syndrome and Prader-Willi syndrome occur. These syndromes have been reported in children conceived with ART, although the rarity of the syndromes and problems generalizing from animal experiments to humans makes a causal relationship difficult to establish. In an Australian study that used data from a national genetics testing laboratory, 37 cases of BeckwithWiedemann syndrome occurred in 1.3 million live births, a rate of one in every 35,580 births [22]. Four of the cases were associated with IVF compared to a matched control group in which one mother had IVF $(P<0.05)$. In a cohort study from the Danish national registries for IVF and for all births, however, no increase in imprinting diseases was linked to ART [23].

\section{Recommendations for action}

Reproductive endocrinologists use a combination of clinical factors to advise couples of the likelihood of success from infertility treatment, and they use clinical tests such basal follicle stimulating hormone levels to further characterize prognosis. Professional practice guidelines are available that recommend transferring fewer embryos among the couples with greatest risk for multiple gestation [24].

Professional guidelines note the elements to be considered when educating patients during the informed consent process for ART [25]. Reproductive endocrinologists routinely counsel patients about the risks from ART, including multiple gestation, ovarian hyperstimulation syndrome, physical injury during procedures, and success rates [26]. It may be prudent to counsel patients about emerging scientific issues as new research is published, such as concerns about embryo culture and ICSI. This would be similar to the approach used for other potential concerns. That is, even though a causal relationship between ART treatment methods and development of ovarian cancer has not been substantiated, the majority of reproductive endocrinologists inform patients of the issue [26].

\section{Effectiveness of interventions}

Little research exists on comprehensive preconception counseling for infertility patients. Studies that have examined counseling about the most common adverse outcome, multiple births, have found that many infertility patients prefer to have twins and sometimes triplets rather than a single infant [27-29]. A randomized trial of a new educational intervention was ineffective at reducing this preference, although other research has found that raising awareness of the risks of multiple births reduces patient preference for multiples [27, 28].

\section{Integration with other preconception services}

Routine preconception counseling for women and men should include information on the many well-documented risk factors for experiencing infertility later in life. When patients who have trouble conceiving present for ART, reinforcing these concepts and combining preconception care at the key decision-making points should help maximize health outcomes for parents and their future children.

\section{References}

1. Stephen EH, Chandra A. Updated projections of infertility in the United States: 1995-2025. Fertil Steril 1998;70:30-4.

2. Rostad B, Schei B, Sundby J. Fertility in Norwegian women: results from a population-based health survey. Scand J Public Health 2006;34:5-10.

3. Gnoth C, Godehardt D, Godehardt E, et al. Time to pregnancy: results of the German prospective study and impact on the management of infertility. Hum Reprod 2003;18:1959-66. 
4. Wright FC, Schieve LA, Reynolds MA, et al. Assisted reproductive technology surveillance-United States, 2002. MMWR Surveill Summ 2005;54:1-24.

5. Toner JP, Grainger DA, Frazier LM. Clinical outcomes among recipients of donated eggs: An analysis of the national experience, 1996-1998. Fertil Steril 2002;78:1038-45.

6. Witsenburg C, Dieben S, Van der Westerlaken L, et al. Cumulative live birth rates in cohorts of patients treated with in vitro fertilization or intracytoplasmic sperm injection. Fertil Steril 2005;84:99_ 107.

7. Hassan MAM, Killick SR. Effect of male age on fertility: Evidence for the decline in male fertility with increasing age. Fertil Steril 2003;79(Suppl 3):1520-7.

8. Neal MS, Hughes EG, Holloway AC, Foster WG. Sidestream smoking is equally as damaging as mainstream smoking on IVF outcomes. Hum Reprod 2005;20:2531-5.

9. Zitzmann M, Rolf C, Nordhoff V, et al. Male smokers have a decreased success rate for in vitro fertilization and intracytoplasmic sperm injection. Fertil Steril 2003;79(Suppl 3):1550-4.

10. Fedorcsak P, Dale PO, Storeng R, et al. Impact of overweight and underweight on assisted reproduction treatment. Hum Reprod 2004; $19: 2523-8$.

11. Frazier LM, Grainger DA, Schieve LA, Toner JP. Follicle stimulating hormone and estradiol levels independently predict success of assisted reproductive technology treatment. Fertil Steril 2004;82:834-40.

12. Strandell A, Lindhard A, Waldenstrom, Thorburn J. Hydrosalpinx and IVF outcome: Cumulative results after salgingectomy in a randomized controlled trial. Hum Reprod 2001;16:2403-10.

13. Adamson D, Baker V. Multiple births from assisted reproductive technologies: A challenge that must be met. Fertil Steril 2004;81:517-22.

14. Reynolds MA, Schieve LA, Martin JA, Jeng G, Macaluso M. Trends in multiple births conceived using assisted reproductive technology, United States, 1997-2000. Pediatrics 2003;111:115962.

15. Papanikolaou EG, Tournaye H, Verpoest W, et al. Early and late ovarian hyperstimulation syndrome: Early pregnancy outcome and profile. Hum Reprod 2005;20:636-41.

16. Kashyap S, Moher D, Fung MKF, Rosenwaks Z. Assisted reproductive technology and the incidence of ovarian cancer: a metaanalysis. Obstet Gynecol 2004;103:785-94.
17. Helmerhorst FM, Perquin DAM, Donker D, et al. Perinatal outcome of singletons and twins after assisted conception: A systematic review of controlled studies. BMJ 2004;328:261. Epub 2004 Jan 23.

18. Aittomaki K, Bergh C, Hazekamp J, et al. Genetics and assisted reproductive technology. Acta Obstet Gynecol Scand 2005;84:46373.

19. Aittomaki K, Wennerholm U-B, Berth C, et al. Should ICSI patients have genetic testing before treatment? A practical proposition to help patient information. Hum Reprod 2004;19:47276.

20. Burello N, Vicari E, Shin P, et al. Lower sperm aneuploidy frequency is associated with high pregnancy rates in ICSI programmes. Hum Reprod 2003;18:1371-6.

21. Arnaud P, Feil R. Epigenetic deregulation of genomic imprinting in human disorders and following assisted reproduction. Birth Defects Res (Part C) 2005;75:81-97.

22. Halliday J, Oke K, Breheny S, Algar EJ, Amor D. BeckwithWiedemann syndrome and IVF: a case-control study. Am J Hum Genet 2004 Sep;75(3):526-8.

23. Lidegaard O, Pinborg A, Andersen AN. Imprinting diseases and IVF: Danish National IVF Cohort Study. Hum Reprod 2005; 20:950-4.

24. SART/ASRM (The practice committee of the Society for Assisted Reproductive Technology and the American Society for Reproductive Medicine). Guidelines on the number of embryos transferred. Fertil Steril 2004;82:773-4

25. ASRM (The Practice Committee of the American Society for Reproductive Medicine). Elements to be considered in Obtaining Informed Consent for ART. 1998, ASRM.

26. Houmard BS, Seifer DB. Infertility treatment and informed consent: Current practices of reproductive endocrinologists. Obstet Gynecol 1999;93:252-7.

27. Ryan GL, Zhang SH, Dokras A, et al. The desire of infertile patients for multiple births. Fertil Steril 2004;81:500-4.

28. Murray S, Shetty A, Rattray A, et al. A randomized comparison of alternative methods of information provision on the acceptability of elective single embryo transfer. Hum Reprod 2004;19:91116.

29. Grobman WA, Milad MKP, Stout J, et al. Patient perceptions of multiple gestations: An assessment of knowledge and risk aversion. Am J Obstet Gynecol 2001;185:920-4. 